\begin{tabular}{|c|l|}
\hline Title & A comparison between Tsallis' s statistics based and generalized quasi-hyperbolic discount models in humans \\
\hline Author(s) & Takahashi, Taiki \\
\hline Citation & $\begin{array}{l}\text { PhysicaA: Statistical Mechanics and its A pplications, 387(2-3), 551-556 } \\
\text { https://doi.org/10.1016/.physa.2007.09.007 }\end{array}$ \\
\hline Issue Date & 2008_01-15 \\
\hline Doc URL & http://hdl.handle.net/2115/51136 \\
\hline Type & article(author version) \\
\hline File Information & Revision1_Takahashi_PHYSICA_A_quasiHyperbolicDiscounting.pdf \\
\hline
\end{tabular}

Instructions for use 


\title{
A comparison between Tsallis' statistics-based and generalized quasi-hyperbolic discount models in humans.
}

\author{
Taiki Takahashi ${ }^{1}$
}

${ }^{1}$ Direct all correspondence to Taiki Takahashi, Unit of Cognitive and Behavioral Sciences, Department of Life Sciences, School of Arts and Sciences, The University of Tokyo, Komaba, Meguro-ku, Tokyo, 153-8902, Japan (taikitakahashi@ gmail.com).

Acknowledgements: The research reported in this paper was supported by a grant from the Grant- in-Aid for Scientific Research ("21st century center of excellence" grant) from the Ministry of Education, Culture, Sports, Science and Technology of Japan, and Yamaguchi endocrinological disorder research grant. 


\section{Summary}

Intertemporal choice has drawn attention in econophysics and neuroeconomics. Both impulsivity and inconsistency in intertemporal choice can be parametrized with a Tsallis' statistics-based q-exponential discount model. Although a recent neuroeconomic study has proposed a dual-self discounting model (a generalized quasi-hyperbolic discounting), no study to date examined the relationship between the q-exponential and quasi-hyperbolic models. We empirically estimated the inconsistency parameter $1-q$ in the q-exponential discount function and the parameter of an internal conflict between dual selves in intertemporal choice by humans. We observed that (i) inconsistency parameter in the q-exponential model was positively related to a conflict between selves within a subject and (ii) q-exponential discounting better fit group data, while quasi-hyperbolic discounting better fit individual data. Our results indicate that (i) inconsistent intertemporal decision-makers may experience strong internal conflict during intertemporal choice behavior, and (ii) the q-exponential and the quasi-hyperbolic discount models should respectively be utilized for group and individual data. The usefulness of the q-exponential and the quasi-hyperbolic in studies of addiction and economic behavior is discussed.

Keywords: Econophysics; Intertemporal choice; Inconsistency; Neuroeconomics; Multiple selves 


\section{Introduction to intertemporal choice models}

Most people prefer an immediate reward to a delayed reward in intertemporal choice (delay discounting) [1-8]. Intertemporal choice behavior is related to addiction, saving behavior, financial planning such as investment and retirement. Therefore, studies in behavioral neuroeconomics, psychopharmacology, behavioral finance, and econophysics have focused on how intertemporal choice is characterized in theoretical and empirical manners. Impulsivity in intertemporal choice refers to preference for small but immediate rewards over large but delayed ones. Consider the following example:

(A) Choose between (A.1) One cup of tea now.

(A.2) Two cups of tea tomorrow.

(B) Choose between (B.1) One cup of tea in one year.

(B.2) Two cups of tea in one year plus one day.

Most people may make impulsive choice in (A) (i.e., (A.1), preference for the small immediate reward); while make patient choice in (B) (i.e., (B.2), preference for the large delayed reward). Therefore, it can be said that most people are patient in discounting in the distant future, but impulsive in the near future, resulting in preference reversal as time passes. Accounting for impulsive and inconsistent choices has been attracting much attention in neuroeconomics [3-9], econophysics and social physics [10-11], because impulsivity and inconsistency in the intertemporal choice behavior are associated with addiction and a number of anomalies in financial markets and saving behavior [1-2].

In order to describe human and animal subject's intertemporal choice behavior, recent econophysical studies [10-11] proposed and examined the following q-exponential discount function based on Tsallis' statistics:

$$
V(D)=A / \exp _{q}\left(k_{q} D\right)=A /\left[1+(1-q) k_{q} D\right]^{1 /(1-q)} \quad \text { (Equation 1) }
$$

where $\exp _{q}()$ is a q-exponential function, $\mathrm{D}$ is a delay until receipt of a reward, $A$ is the value of a reward at $D=0$, and $k_{q}$ is a parameter of impulsivity at delay $D=0$ (q-exponential discount rate). Note that when $q=0$, equation 1 is the same as a hyperbolic discount function (i.e., $V(D)=A /(1+k D)$ ) [1-2], while $q \rightarrow 1$, is the same as an exponential discount function (i.e., $V(D)=A \exp (-k D)$ ) [1-2]. It is to be noted that in 
exponential discounting ( $\mathrm{q} \rightarrow 1$ in equation 1 ), preference reversal never occurs, because the discount rate: $=-(d V / D) / V=k_{q}$ is time-independent when $\mathrm{q} \rightarrow 1$. The dynamics of a discount rate will more extensively be discussed later. We have previously shown that the q-exponential discount function is capable of continuously quantifying human subjects' inconsistency in intertemporal choice [11]. Namely, human agents with smaller $\mathrm{q}$ values are more inconsitent in intertemporal choice. If $q$ is less than 0 , the intertemproal choice behavior is more inconsistent than hyperbolic discounting. Additionally, 1- $q$ can be utilized as an inconsistency parameter.

Alternatively, behavioral economic studies have proposed that the inconsistency in intertemporal choice may be attributable to an internal conflict between "multiple selves" within an agent [12]. This hypothesis states that (a) there are at least two exponential discounting selves (i.e., two exponential discount rates) in a single human individual and (b) when delayed rewards are at the distant future ( $>1$ year), a self with a smaller discount rate wins; while delayed rewards approach to the near future (within a year), a self with a larger discount rate wins, resulting in preference reversal over time [1,12]. This intertemporal choice behavior has been referred to as quasi-hyperbolic discounting [1,12]. Furthermore, a recent neuroeconomic study utilizing functional magnetic resonance neuroimaging proposed a generalized quasi-hyperbolic discount model in which there are linearly weighted dual selves in intertemporal choice [13]. More specifically, the study proposed the following linearly-weighted two-exponential functions as generalized quasi-hyperbolic discounting:

$$
V(D)=A\left[w \exp \left(-k_{1} D\right)+(1-w) \exp \left(-k_{2} D\right)\right]
$$

(Equation 2)

where $0<w<1$ is a weighting parameter and $k_{1}$ and $k_{2}$ are two exponential discount rates $\left(k_{1}<k_{2}\right)$. Note that a larger exponential discount rate of the two $\left(k_{2}\right)$ corresponds to an impulsive self; while a smaller discount rate $k_{1}$ corresponds to a "patient self". The mentioned neuroeconomic study observed that, within a single subject, "impulsive self " (a larger discount rate $k_{2}$ ) was associated with the neural activities in reward-processing midbrain regions; while "patient self" $\left(k_{l}\right)$ was associated with those in prefrontal brain regions [13]. It is to be noted that in the neuroimaging study, the generalized quasi-hyperbolic model was originally proposed for a discrete-time discount function. I have here extended it into a continuous-time discount function. Although the psychological interpretation of inconsistent intertemporal choice behavior in the quasi-hyperbolic discounting is intuitively intriguing, no study to date examined the 
relationship between the (generalized) quasi-hyperbolic discount model and the q-exponential discount model in an empirical manner.

\section{Discount rates in q-exponential and quasi-hyperbolic discounting}

Because a discount rate and its dynamics are important, I now summarize the properties of discount rates in the q-exponential and the generalized quasi-hyperbolic discount models. It is important to note that in any continuous time-discounting functions, a discount rate is defined as $-(d V(D) / d D) / V(D)$, independently of functional types of discount models, and larger discount rates indicates more impulsive intertemporal choice. In the q-exponential discount model, the discount rate is:

$$
\text { (q-exponential discount rate) }=k_{q} /\left(1+k_{q}(1-q) D\right) . \quad \text { (Equation 3) }
$$

We can see that when $q=1$, the discount rate is independent of delay $D$, corresponding to exponential discounting (consistent intertemporal choice); while for $q<1$, the discount rate is a decreasing function of delay $D$, resulting in preference reversal. This can be checked by calculating the time-derivative of the q-exponential discount rate:

$(d / d D)(\mathrm{q}-$ exponential discount rate $)=-k_{q}^{2}(1-q) /\left(k_{q}(1-q) D+1\right)^{2} \quad$ (Equation 4)

which is negative for $q<1$. Also, impulsivity at delay $\mathrm{D}=0$ is equal to $k_{q}$ irrespective of $q$. For the generalized quasi-hyperbolic discount model (Equation 2), the discount rate is:

$\left.\left[k_{2}(1-w) \exp \left(-k_{2} D\right)+k_{1} w \exp \left(-k_{1} D\right)\right] /(1-w) \exp \left(-k_{2} D\right)+w \exp \left(-k_{1} D\right)\right] . \quad$ (Equation 5)

It is to be noted that at delay $D=0$, the generalized quasi-hyperbolic discount rate $=$ $w k_{1}+(1-w) k_{2}$. This is consistent with the intuitive interpretation of the quasi-hyperbolic model that impulsivity corresponds to linearly-weighted discount rates. Furthermore, a time-derivative of the discount rate, i.e., $(d / d D)$ (generalized quasi-hyperbolic discount rate) is:

$-w(1-w)\left(k_{2}-k_{1}\right)^{2} \exp \left(k_{2} D+k_{1} D\right) /\left[w \exp \left(k_{2} D\right)+(1-w) \exp \left(k_{1} D\right)\right]^{2}, \quad$ (Equation 6)

which is negative because $1-w>0$. This also indicates that the discount rate is a decreasing function of delay. Psychologically, it is supposable that the weighted 
difference between discount rates $k_{2}>k_{1}$ :

conflict parameter:= $(1-w) k_{2}-w k_{1}$

(Equation 7)

may indicate the strength of "internal conflict" between impulsive and patient selves in intertamporal choice. The reason is that this parameter indicates the degree of disagreement between weighted impulsive and patient selves in intertemporal choice. No study to date examined the relationship between the conflict parameter and the q-parameter in the q-exponential discount model, i.e., an inconsistency parameter. To examine the relation between conflict parameter $(1-w) k_{2}-w k_{1}$ and inconsistency parameter $(1-q)$ in the two models was a part of the objective of the present study.

\section{Behavioral data}

\subsection{Delay discounting task and data analysis methods}

I conducted a behavioral experiment assessing subjects' intertemporal choice behavior by adopting a similar procedure of previous studies $[4,14,15]$. (the sum of the reward was 100,000 yen, with 7 delays ranging from 1 week to 25 years). Subjects were students of the University of Tokyo $(\mathrm{N}=20)$. In order to exclude the psychopharmacological influences of nicotine dependence on discounting behavior [4], only non-smokers were employed.

Firstly, participants were seated individually in a quiet room, and face the experimenter across a table. After that, participants received the simple instruction that the monetary reward in this experiment was hypothetical, but the experimenter wanted you to think as though it were real money. Then the participants were asked to choose between the card describing money delivered immediately and the card describing money delivered after a certain delay. The left card viewed from participants indicated the amounts of money that could be received immediately (or certainly, in a probability discounting task), and the right card indicated 100,000 yen that could be received after a certain delay. Monetary rewards and the delay were printed on $3 \times 5$ index cards. The 27 monetary reward amounts were 100,000 yen (about $\$ 1,000$ ), 99,000 yen, 96,000 yen, 92,000 yen, 85,000 yen, 80,000 yen, 75,000 yen, 70,000 yen, 65,000 yen, 60,000 yen, 55,000 yen, 50,000 yen, 45,000 yen, 40,000 yen, 35,000 yen, 30,000 yen, 25,000 yen, 20,000 yen, 15,000 yen, 10,000 yen, 8,000 yen, 6,000 yen, 4,000 yen, 2,000 yen, 1,000 yen, 500 yen, and 100 yen. The seven reward delays were 1 week, 2 weeks, 1 month, 6 months, 1 year, 5 years, and 25 years. The experimenter turned the 27 "immediate" 
cards sequentially. The card started with 100,000 yen, down to 100 yen, and back to 100,000 yen. For each card, the participant pointed the immediate or delayed reward. The experimenter wrote down the last immediate reward chosen in the descending order, and the first immediate reward chosen in the ascending order, and the average of them was used as the point of subjective equality (hereafter indifference point) in the following analysis.

After the behavioral experiment, parameter estimation was conducted. By utilizing nonlinear curve fitting (delay and indifference point are independent and dependent variables, respectively) procedure of R statistical language (http://www.r-project.org/), subjects' group median and individual parameters in intertemporal choice (i.e., $k_{q}$ and $q$ values in the q-exponential discount model, and $k_{1}, k_{2}, w$ in the generalized quasi-hyperbolic model) were estimated. Also, in order to examine the goodness-of-fit for the two models, I computed AIC (Akaike Information Criterion) values for each model at both group and individual levels. It is important to note that smaller AIC corresponds to better fitting [14]. In order to compare AICs between the q-exponential and the generalized quasi-hyperbolic models at the individual level, we employed Student's t-test, because Kolmogolov-Smirnov test did not reveal a significant deviation from a normal distribution $(p>0.05)$, following the previous study [14]. Finally, I examined the relationship between individual differences in inconsistency and internal conflict in intertemporal decision-making. In order to exclude the influences of outlier values, Pearson's product moment correlation coefficient was calculated after log-transformation, following the established procedure in discounting literature [15]. Significance level was set at 0.05 throughout.

\subsection{Parameters in q-exponential and generalized quasi-hyperbolic models}

The analysis of AIC values indicated that the q-exponential discount model better fit group median data; while the generalized quasi-hyperbolic discount function significantly better fit the individual data ( $\mathrm{t}$-test, $p<0.05)$. The estimated parameter values are presented in Table 1.

\subsection{Relationship between q-exponential and generalized quasi-hyperbolic models}

I observed that there was a significant positive correlation between the inconsistency parameter in the q-exponential discount model (i.e., 1- $q$ in Equation 1) and the conflict parameter in the generalized quasi-hyperbolic discount model (i.e., $(1-w) k_{2}-w k_{1}$, Equation 7). A scatterplot of the logged parameters are presented in Fig.1. The positive correlation indicates that more inconsistent subjects are more susceptible to 
internal conflict between impulsive and patient selves, when performing intertemporal decision.

With respect to consistency and an internal conflict between selves in intertempora choice, the group median $q$ in Equation 1 (consistency) and $w$ in Equation 2 (a weight of dual selves) were smaller than zero and close to 0.5 , respectively. These findings imply that representative human intertemporal choice is more inconsistent in comparison to hyperbolic discounting, which corresponds to $q=0$, and under a strong internal conflict (note that smaller $q$ values correspond to more inconsistent discounting, and the internal conflict is maximal at $w=0.5$, and minimal at $w=0$ and 1$)$. It should also be noted that estimated values of $k_{2}$ was significantly larger than those of $k_{1}(p<0.01)$, indicating that the dual-self exponential discounting (quasi-hyperbolic discounting) significantly better describes human intertemporal choice, in comparison to simple exponential discounting.

\section{Conclusions and implications}

The present empirical examination of the q-exponential discount function and the generalized quasi-hyperbolic discount model has demonstrated that (i) the q-exponential discount function better fit group data; while the generalized quasi-hyperbolic discount function better fit the individual data, and (ii) inconsistency in the q-exponential discounting and "internal conflict between selves" in the quasi-hyperbolic discounting are positively associated. This study is the first to establish a continuous-time version of the generalized quasi-hyperbolic discount function (Equation 2) and examine the relationship between the q-exponential and the generalized quasi-hyperbolic discount models.

The present findings have several implications for future studies in neuroeconomics, neuropsychopharmacology, and econophysics. Future neuroeconomic studies should examine (i) neural correlates of inconsistency in intertemporal choice (i.e., 1-q) by utilizing the q-exponential discount model and (ii) whether the inconsistency is associated with the relatively higher activation of reward-processing midbrain regions, compared to the prefrontal cortical activation. When neuropsychophamacological studies on addiction examine a group difference in discounting behavior between healthy controls and drug-dependent subjects, and/or between a control and a neuropharmacological administration groups, it is recommendable that the q-exponential discount function should be employed; on the other hand, in psychopharmacological and econophysical investigations into individual 
differences in discounting behavior (e.g., addiction and saving) and/or correlation between neurochemical substrates such as cortisol, testosterone and nicotine [4-6] and individual discounting behavior, the generalized quasi-hyperbolic discount model should be employed, The reason is that the present study demonstrated that the q-exponential discount model better fit the group data; while the generalized quasi-hyperbolic model better fit the individual data. 


\section{References}

[1] Frederick, S., Loewenstein, G., \& O’Donoghue, T. Time discounting and time preference: A critical review. Journal of Economic Literature, 40, (2002)351-401.

[2] Ainslie G.. Precis of Breakdown of Will. Behav Brain Sci. 2005 28: 635-650

[3] Takahashi T. Loss of self-control in intertemporal choice may be attributable to logarithmic time-perception. Med Hypotheses. 2005;65(4):691-693.

[4] Ohmura Y, Takahashi T, Kitamura N. Discounting delayed and probabilistic monetary gains and losses by smokers of cigarettes. Psychopharmacology (Berl). 2005 182:508-515.

[5] Takahashi, T. Cortisol levels and time-discounting of monetary gain in humans. Neuroreport, 15 (2004) 2145-2147.

[6] Takahashi T, Sakaguchi K, Oki M, Homma S, Hasegawa T. Testosterone levels and discounting delayed monetary gains and losses in male humans. NeuroEndocrinol Lett. 27(4):439-444 2006

[7] Takahashi T. Time-estimation error following Weber-Fechner law may explain subadditive time-discounting. Med Hypotheses. 2006;67(6):1372-1374.

[8] McClure SM, Laibson DI, Loewenstein G, Cohen JD. Separate neural systems value immediate and delayed monetary rewards. Science. 2004 306(5695):503-507.

[9] Lee D. Neuroeconomics: making risky choices in the brain. Nature Neuroscience. 2005 8(9):1129-1130.

[10] Cajueiro D.O. A note on the relevance of the q-exponential function in the context of intertemporal choices. Physica A 364 (2006) 385-388.

[11] Takahashi T, Oono H, and Radford MHB, Empirical estimation of consistency parameter in intertemporal choice based on Tsallis' statistics. Physica A (in press)

[12] Laibson, D., 1997. Golden eggs and hyperbolic discounting. Quarterly Journal of 
Economics 112, 443-477.

[13] McClure SM, Ericson KM, Laibson DI, Loewenstein G, Cohen JD. Time discounting for primary rewards. J Neurosci. 2007 27:5796-5804.

[14] Takahashi T, Oono H, Radford MHB. Comparison of probabilistic choice models in humans. Behavioral and Brain Functions 2007, 3:20

[15] Ohmura Y, Takahashi T, Kitamura N, Wehr P. Three-month stability of delay and probability discounting measures. Exp Clin Psychopharmacol. 2006 14(3):318-328. 
Table 1 a. Parameters in intertemproal choice for group median data at each delay

\begin{tabular}{lllll}
\hline$q$-exponential & $q$ & \multicolumn{2}{c}{$k_{q}$} & \multicolumn{1}{c}{ AIC } \\
& -3.8699299 & 0.0019852 & 116.187 \\
\hline Generalized & $k_{1}$ & $k_{2}$ & $w$ & AIC \\
quasi-hyperbolic & $4.439 \times 10^{-5}$ & $3.07 \times 10^{-03}$ & 0.59 & 121.437 \\
\hline
\end{tabular}

Consistency parameter q was smaller than 1. q-exponential discount function better fit group data. Note that smaller AIC corresponds to better fitting.

Table $1 \mathrm{~b}$. Parameters in intertemproal choice for individual data

\begin{tabular}{lllll}
\hline$q$-exponential & $q$ & \multicolumn{2}{l}{$k_{q}$} & \multicolumn{1}{l}{ AIC } \\
& $-3.8699 \pm 7.9$ & $0.0105 \pm 0.02$ & $132.92 \pm 9.0$ \\
\hline Generalized & $k_{1}$ & $k_{2}$ & $w$ & $A I C$ \\
quasi-hyperbolic & 0.0003083 & 0.019 & 0.625 & 129.35 \\
& \pm 0.0011 & \pm 0.022 & \pm 0.31 & \pm 11.1 \\
\hline
\end{tabular}

Consistency parameter q was smaller than 1. quasi-hyperbolic discount function better fit individual data. Data are expressed as Mean \pm Standard Deviation. Note that smaller AIC corresponds to better fitting. 
Fig. 1

Scatterplot of logged inconsistency and conflict parameters. A significant positive correlation was observed $(r=0.45, p<0.05, \mathrm{~N}=20)$.

Logged (1-q) : Inconsistency

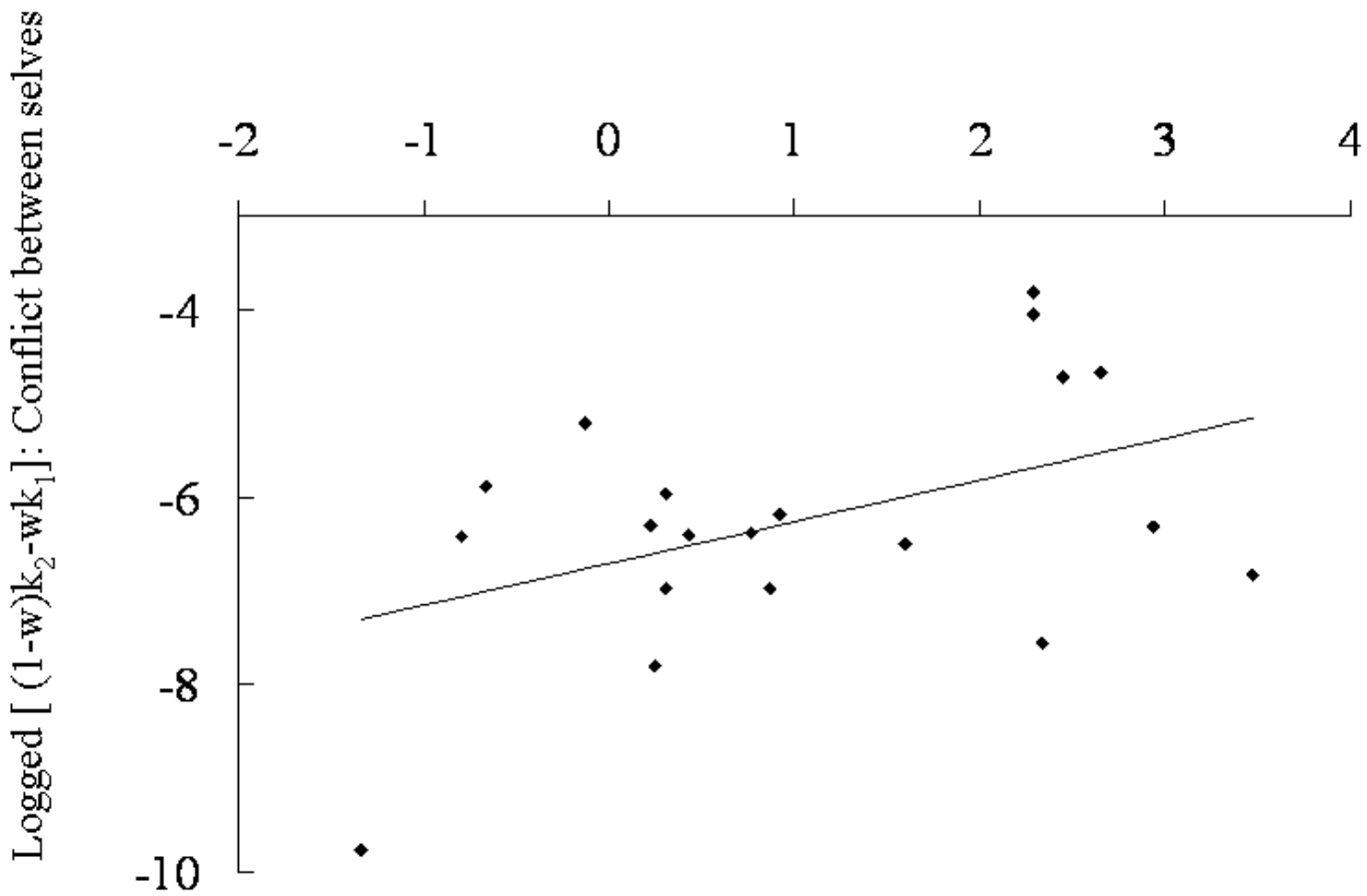

